\title{
Barbara Cassin - « Il faut au moins deux langues pour savoir qu'on en parle une »
}

Entretien avec Fabienne Durand-Bogaert

\section{Fabienne Durand-Bogaert}

\section{OpenEdition}

\section{Journals}

Édition électronique

URL : http://journals.openedition.org/genesis/1294

DOI : $10.4000 /$ genesis. 1294

ISSN : 2268-1590

Éditeur :

Presses universitaires de Paris Sorbonne (PUPS), Société internationale de génétique artistique littéraire et scientifique (SIGALES)

\section{Édition imprimée}

Date de publication : 15 avril 2014

Pagination : 129-137

ISBN : 9782840509370

ISSN : 1167-5101

\section{Référence électronique}

Fabienne Durand-Bogaert, « Barbara Cassin - « II faut au moins deux langues pour savoir qu'on en parle une » », Genesis [En ligne], 38 | 2014, mis en ligne le 29 juin 2016, consulté le 11 juin 2020. URL: http://journals.openedition.org/genesis/1294; DOI : https://doi.org/10.4000/genesis.1294 


\title{
Barbara Cassin " Il faut au moins deux langues pour savoir qu'on en parle une »
}

\author{
Entretien avec Fabienne Durand-Bogaert
}

Fabienne Durand-Bogaert - J'aimerais que vous retraciez pour les lecteurs de notre revue la genèse de cette entreprise $d u$ Vocabulaire européen des philosophies ${ }^{1}$. Pourriez-vous décrire le cheminement de la réflexion qui vous a menée à la naissance de ce projet?

Barbara Cassin - C'est étrange comme, quand quelqu'un vous pose une question de genèse, on s'aperçoit qu'on a affaire à l'oubli. Cela fait maintenant presque dix ans que le dictionnaire est sorti, et j'avais commencé à y penser quinze ans avant sa sortie. La problématique de la traduction me vient évidemment de mon rapport à la philologie, qui est mon cœur de métier, si j’ose dire, en tant que spécialiste de philosophie grecque ancienne. La traduction, en philosophie grecque, m'est toujours apparue comme le point ultime de toute la série d'opérations qui permettent d'établir un texte : il s'agit de «dé-déformer » sans arrêt. Dans le cas de la sophistique, j'ai l'habitude de dire que c'est une sorte de paléontologie de la perversion : on hérite de quelques os qui ont été mâchouillés par les ontologistes, qui nous les ont transmis de telle manière qu'ils ne ressemblent plus du tout à des os de dinosaures.

La traduction constitue donc l'aboutissement d'une série d'opérations sur le texte, qui impliquent en quelque sorte sa digestion. Or ce qui apparaît quand on commence par l'opération finale, c'est-à-dire par la traduction, c'est que les traductions sont multiples et qu'elles ont toutes un sens. Par «sens », j'entends aussi une intention. Heidegger traduisant Parménide, ce n'est pas Jonathan Barnes traduisant Parménide. L'intention n'est pas la même, le texte n'est pas le même ; le Parménide de Heidegger et le Parménide de Barnes ne sont pas un seul et même Parménide. C'est évidemment vrai lorsqu'on traduit de la poésie. Or - et ce point m'a sauté aux yeux à partir de la philosophie grecque - c'est vrai aussi pour la traduction de la philosophie. On peut dire que la traduction est une création littéraire, une création de langue, une création de langue dans une langue. Dans le domaine littéraire, cela est admis et reconnu. Mais cette dimension de création dans la traduction vaut tout autant en philosophie, même si elle est bien moins reconnue, voire complètement méconnue.

Le dictionnaire prend place dans un double cadre : celui de la construction de l'Europe d'une part, celui $\mathrm{du}$ rapport entre philosophie analytique et philosophie herméneutique d'autre part. Ce qui m'intéressait, dans ce cadre, c'était de montrer qu'il fallait compliquer l'universel. Et qu'on ne pouvait pas imaginer que nous philosophions par concepts sans penser aussi, et peut-être d'abord, que nous philosophions en mots. J'ai l'habitude de caractériser le projet (par exemple dans la «Présentation » du dictionnaire) comme un geste politique et philosophique, qui refuse tant le « nationalisme ontologique » que le globish. Refuser le globish, voilà le geste politique : il ne s'agit pas de réduire toutes les langues à une langue de communication qui n'en est plus une, qui n'a d'ailleurs rien à voir avec l'anglais, ce dont le British Council se désole tous les jours. La langue n'existe pas. Comme dit Humboldt, il n'y a pas le langage, il y a des langues. Et une langue est autre chose qu'un vecteur de communication. C'en est un, mais c'est aussi autre chose.

1. Vocabulaire européen des philosophies : dictionnaire des intraduisibles, dir. Barbara Cassin, Paris, Éditions du Seuil/Le Robert, 2004. 
F. D.-B. - Quand vous dites que la langue n'existe pas, vous voulez dire en essence?

B. C. - Je veux dire que le langage n'existe pas : comme entité unique, Humboldt ne l'a jamais rencontré, moi non plus. Le grec dit que l'homme est un animal ou un vivant doué de logos. Sous logos, il faut entendre « langue », « langage », « raison », c'est donc un terme profondément équivoque - une équivoque caractéristique de la langue grecque. On en arrive tout de suite à l'un des motifs contemporains qui animent le projet du dictionnaire : « une langue, entre autres, n'est rien de plus que l'intégrale des équivoques que son histoire y a laissé persister ». C'est une phrase de Lacan dans L'Étourdit, qui caractérise à mes yeux toute langue, pas seulement les langues de l'inconscient, en tant qu'elle est une, et une parmi d'autres.

F. D.-B. - Une autre question très concrète : j'aimerais savoir comment vous avez réuni votre équipe. Comment avez-vous procédé pour vous associer tous ces chercheurs, je crois qu'il y en avait à peu près cent cinquante, philosophes pour la plupart, mais aussi linguistes, spécialistes de langues anciennes, historiens de l' histoire et d'autres disciplines scientifiques?

B. C. - Ce sont presque tous des compagnons de route. Il arrive aussi que l'on rencontre des compagnons en route, mais disons que je suis partie d'un noyau d'amis philosophes avec lesquels je savais qu'il était possible de travailler. Je dois revenir ici à ce que j'entends par, ou à ce qui se cache sous, le terme de "nationalisme ontologique » : Heidegger. J'étais au séminaire de Thor, avec Heidegger et René Char, et j'ai reçu une formation liée, d'une manière ou d'une autre, aux problématiques heideggériennes. En même temps, j'ai été voir ailleurs, auprès de professeurs comme Jean Bollack et Heinz Wismann. C'est cette tension qui m'a appris à réfléchir aux traductions. De bons historiens de la philosophie, de ceux qui savent bien lire les textes, j'en avais donc rencontrés au cours de l'une ou l'autre de ces formations. Ensuite, j'ai naturellement réfléchi aux compétences dont nous avions besoin : compétences linguistiques, compétences disciplinaires dans des domaines différents de la philosophie. C'est comme cela que j'ai réuni cette équipe. Très simplement. J'ai expliqué le projet à un tout petit nombre d'amis, comme Jean-François Courtine, Charles Baladier, avec qui j'avais déjà travaillé et qui a joué un très grand rôle ; Alain de Libera, Charles Malamoud... Nous avons fait une première réunion tout à fait informelle, chez moi, pour réfléchir. J'ai essayé d'expliquer ce que je voulais faire. On retombait toujours dans l'histoire de la pensée, dans le Ritter. J'essayais d'expliquer pourquoi je n'en voulais pas. Le Ritter, pour moi, c'est un monument de nationalisme ontologique : il y a deux langues philosophiques, le grec et l'allemand (qui est plus grec que le grec), et puis on peut faire des incursions dans des langues qui, tout d'un coup, à une époque donnée, se trouvent au plus près de l'être. Au moment de la Renaissance, c'est l'italien, par exemple. Il s'agit toujours d'une hiérarchie des langues, de sorte qu'on ne philosophera jamais en français, sauf à un tout petit moment, celui de Descartes, et peu en anglais, sauf au moment de Locke et Hume. Je voulais échapper à la caricature de cette conception. Je pense moi aussi que le grec et l'allemand sont des langues fondamentales en philosophie, mais je crois que ce n'est pas de cette sacralisation que nous avons besoin, bien au contraire.

Tullio Gregory qui était présent à cette réunion m'a posé la question : "Barbara, vos entrées, ce sont des concepts ou des mots? » J'ai répondu : ce sont des mots, et tout s'est éclairé alors pour moi-même. Après quoi, j'ai cherché les forces qui manquaient. Par exemple, Heinz Wismann m'a présenté Constantin Sigov, qui a réuni une équipe pour travailler sur l'ukrainien et le russe. Fernando Santoro est venu du Brésil pour travailler avec moi sur les Présocratiques, et je l'ai enrôlé dans notre projet, il a écrit lui-même un certain nombre d'articles sur le portugais. C'est ainsi que les choses se sont passées.

Pour concevoir le dictionnaire, le mettre en page, l'inventer formellement, j'ai eu la chance d'avoir l'aide de Gonzague Raynaud, au Robert, qui a su trouver la forme appropriée au projet en comprenant de l'intérieur la différence entre les types d'entrées, les hiérarchies, les exemples, les encadrés, les renvois.

Le processus a été très long, pour plusieurs raisons, et notamment parce qu'au Seuil, il y avait un préparateur de copie extraordinaire, sans lequel rien ne pouvait se 
faire, et qui a été indisponible pendant environ deux ans. Vous savez, c'est un livre dans lequel il ne peut y avoir que des fautes, ce qui le rend extrêmement complexe à réaliser et à relire... Dans chaque article, ce préparateur repérait des dizaines de fautes. Il connaissait toutes les langues, y compris l'hébreu et l'arabe. Sans lui, je n'avais pas le courage de porter le livre à l'impression. J'ai donc attendu qu'il soit disponible à nouveau. Cela a pris du temps, d'autant que je traversais moi-même des moments difficiles, la maladie et le décès d'un tout proche. Le dictionnaire était ce qui me faisait tenir, mais avec très peu de temps à y consacrer.

F. D.-B. - Comment les contributeurs ont-ils procédé, leur avez-vous demandé de traiter spécifiquement une entrée? Les propositions sont-elles venues d'eux, ont-elles été faites en commun?

B. C. - Nous avons été invités à Naples, à l'Institut italien pour les études philosophiques, par Gerardo Marotta, avec Yves Hersant. Nous y avons tenu une première réunion générale, où étaient présentes toutes les personnes que j'avais envie d'associer à ce projet, et c'est là que nous nous sommes réparti les tâches, d'abord par grands domaines. Nous avons choisi des « directeurs de champ »: Jean-François Courtine pour l'objectivité, Étienne Balibar pour la subjectivité, Philippe Raynaud pour le droit et la loi, Marc Crépon, rejoint par Marc de Launay, pour l'éthique, Jacqueline Lichtenstein pour l'esthétique, Alain de Libera, avec Irène Rosier-Catach et moi-même, pour le langage et la logique, etc. Chacun a proposé les points qui, dans sa vie de philosophe et dans son expérience de traducteur, l'avaient arrêté. Par exemple, lorsqu'il avait eu envie de mettre une note de bas de page, ou lorsqu'il avait rencontré une note décisive. Les notes de bas de page des traducteurs sont devenues texte principal.

Chacun, dans le domaine qui lui revenait, a proposé des mots en langues, à partir de textes essentiels. Sur cette base, nous avons fait ce qui était faisable, ce qui était à notre portée, à nous tous, en étendant le «nous » à des praticiens d'autres langues - l'ukrainien, le portugais, l'espagnol par exemple, alors que pour l'italien, le grec, le latin, l'arabe, l'hébreu, l'allemand, nous avions des spécialistes dans l'équipe. Chacun de nous avait ses idées sur le corpus de textes, donc sur les mots, à prendre en compte, et savait à qui il pouvait s'adresser. Nous avons réuni toutes nos compétences et j'ai cherché les équilibres. Nous avons tenté de fabriquer un premier « modèle », avec l'article Moment, Françoise Balibar pour le momentum en physique, Philippe Büttgen pour der/das Moment, et moi pour le kairos grec. Chaque directeur de champ devait lui-même se charger d'un certain nombre d'entrées pour donner le ton général et pour structurer la chose. Ces premières entrées, je dois dire qu'il a fallu presque à chaque fois les réécrire ; réfléchir sur ce qui avait été fait et comprendre pourquoi ça n'allait pas. Nous avons tous tendance à ne pas tenir suffisamment compte de la différence des langues, à ne pas vouloir partir de là, et à prendre l'histoire de la pensée comme point de départ, une fois de plus. Je crois que pas un article, parmi les premiers, n'est passé tel quel. Par la suite, le pli s'est pris.

F. D.-B. - Vous avez vous-même rédigé un encadré sur la question de l'unheimlich chez Freud, qui figure sous l'entrée Heimat. Tel semble être le principe d'organisation du dictionnaire : vous créez une sorte de réseau sémantique, étymologique. Comment fonctionne-til exactement?

B. C. - Il y a plusieurs types d'articles. Certains ont une entrée dans une seule langue, comme c'est le cas pour Heimat (et pour l'encadré unheimlich, le lien est étymologique en effet). D'autres ont une entrée plurilingue, comme Mind - Geist - esprit, pour reprendre un exemple bien connu; et c'est souvent une comparaison de réseaux, par exemple «Law/Right, loi/droit »- la coupure ne passe pas du tout au même endroit dans les deux langues, et cela ne va pas sans produire des quiproquos européens.

Il y a des entrées « recouvertes » par le français, où le français sert de métalangue, par exemple Aimer. Le français aimer confond, sur le modèle du latin, sous un vocable unique diverses manières d' « aimer en langues » : aussi bien philê̂n, agapân, erân que to love, to like, que nous explorons alors.

Tous les lemmes sont liés par des systèmes de renvois et par des entrées, dites «directionnelles », 
rédigées par moi pour servir d'aiguillages pour renvoyer aux lemmes. Par exemple, l'entrée Malaise renvoie à des termes qui disent les dysfonctionnements du rapport âme-corps, les types de souffrance en jeu individuelle, ontologique, nationale -, et dirige alors vers des mots comme acedia, desengaño, saudade, dor, angoisse, mélancolie, spleen, nostalgie ou Sehnsucht.

Il y a également des entrées «d'ordre 2 », sorte de méta-entrées, qui prennent en compte un problème dans son entier (comme le genre des noms, l'ordre des mots, le temps et l'aspect), ou une langue dans son tout. Ces dernières sont les entrées les plus difficiles, car les plus susceptibles d'en revenir à un nationalisme ontologique. Pour l'éviter, nous avons toujours essayé de lier un problème et une langue. On peut avoir une entrée sur l'allemand en général, il y en a même deux, mais l'une d'entre d'elles par exemple observe comment les particules interviennent dans la formation des mots - je voulais l'appeler "Métaphysique des particules ». Ce n'est pas tant une entrée sur l'allemand comme langue, que sur un trait spécifique d'où peuvent découler des singularités philosophiques sensibles de la langue allemande. De même, pour le russe, nous avons une entrée "Diglossie », pour explorer la différence entre «langue haute » et "langue basse », sans laquelle on ne comprend pas les textes russes.

Surtout, j'ai tenté de faire des contrepoints dans une entrée. Par exemple quand une entrée était rédigée par un heideggérien « dur », j'ai proposé une autre vision d'un même texte, une autre écoute de l'étymologie, un autre effet de langue : ainsi du rapport entre l'entrée Heimat et l'encadré unheimlich, coup de projecteur hétérogène, que vous évoquiez.

F. D.-B. - D'où l'importance aussi de la présentation, de la forme éditoriale que vous lui avez donnée. Avec le recul, trouvez-vous qu' il y ait des lacunes, des choses dont vous déplorez aujourd' hui l'absence?

B. C. - Il n'y a que ça ! Ma seule justification, c'est que ce dictionnaire est un geste philosophique, et pas une œuvre achevée. C'est une energeia et non un ergon, exactement comme une langue. D'autre part, les entrées choisies sont des symptômes des différences des langues. Ce ne sont pas du tout les différences des langues, ni même non plus les points essentiels des différences des langues. Comme je l'ai dit, nous avons fait ce que nous étions capables de faire à ce moment-là.

F. D.-B. - Je suppose que cette entreprise du dictionnaire a généré de l'archive, et même une archive assez colossale. Où se trouve-t-elle? Dans les ordinateurs de chacun, ce qui la rend susceptible de se perdre? Avezvous songé à la déposer?

B. C. - Je ne l'ai pas déposée. Il y a eu une archive papier que j'avais en ma possession. Je ne crois pas l'avoir conservée, car elle était très volumineuse et générait des confusions. J'ai, dans de vieux ordinateurs qui n'existent plus et sur des disquettes qu'on ne sait plus ouvrir, l'archive des différentes versions, c'est tout. Je dois dire que l'un des pires moments a été quand nous avons fabriqué des textes avec la fonction «suivi des modifications ». Cela a eu des résultats terribles à l'édition : des pans entiers ont disparu, ce dont nous ne nous sommes pas aperçus tout de suite.

F. D.-B. - On peut imaginer que cette archive aurait pu devenir un outil de consultation pour les chercheurs qui s'intéressent à la critique génétique.

B. C. - Il aurait fallu que l'on se voie il y a vingt-cinq ans ! [rires]

F. D.-B. - Revenons sur l'esprit même de l'entreprise. Je voudrais mettre en rapport votre démarche avec cette remarque faite par Schleiermacher dans son texte consacré aux méthodes du traduire, où il explique que chaque homme, pour une part, est dominé par la langue qu'il parle, qu'il est dans la violence de la langue (in der Gewalt der Sprache). L'homme et sa pensée sont un produit de la langue, et on ne peut rien penser en dehors de cette limite. On a là la description d'une sorte de déterminisme d'ordre linguistique. Est-ce que convoquer plusieurs langues, comme le fait le Vocabulaire, voire parler plusieurs langues, est une manière d'échapper à ce déterminisme? 
$B$. C. - Oui. C'est une manière d'y échapper en en prenant conscience : dès qu'on en prend conscience, on y échappe, en tout cas ce n'est plus le même type de violence. Je rapprocherais cela de ce que dit Derrida : une langue, ça n'appartient pas.

F. D.-B. - Pourtant, on parle de «posséder » une langue.

B. C. - Oui, mais une langue, ça n'appartient pas ; donc on n'appartient pas non plus à une langue. Je suis en train de travailler à un petit livre sur la nostalgie. Les étapes en sont Ulysse, Énée, Arendt, Günther Anders. Qu'est-ce qui se passe quand on a pour patrie sa langue ? Cela ne fonctionne, dans le cas de Hannah Arendt, que dans la mesure où elle refuse toute confusion entre la langue et le peuple. Je me demande s'il peut y avoir la violence dont vous parlez quand il n'y a plus cette collusion, quand une langue, ça n'appartient pas. Au fond, ce dictionnaire est là pour expliquer comment une langue, ça n'appartient pas. Mon idée, c'est qu'il faut au moins deux langues pour savoir qu'on en parle une. Quand on sait que c'est une langue que l'on parle, la violence n'est déjà plus la même. On sait que c'est une langue et qu'il y en a d'autres : « une langue entre autres ». On commence à pouvoir la comparer, réfléchir sur comment elle est fabriquée, et quel monde elle produit. Alors, à mon avis, on échappe à la violence aveugle. Or la violence, me semble-t-il, n'est vraiment violence que quand elle est aveugle. Je ne dis pas qu'on échappe à sa langue, qu'on va cesser de rêver dans sa langue, ou d'avoir envie de parler ou d'écrire dans sa langue. Je ne dis pas que toutes les langues vont revenir au même. Mais on échappe à cette violence, disons, ontologico-politique.

F. D.-B. - Il me semble que la phrase de Schleiermacher décrit la violence de l'emprise, sur nous, de la langue maternelle.

B. C. - Mais Schleiermacher fait un geste analogue à celui du dictionnaire des intraduisibles. Il fait prendre conscience qu'on est sous l'emprise de la langue, et que c'est par cette conscience qu'on peut commencer à mettre l'emprise en perspective, donc à y échapper. À traduire. Pour moi, et pour
Schleiermacher aussi sans doute, l'emprise de la langue, c'est la vision du monde qu'elle implique. Mon travail sur le dictionnaire n'aurait pas été possible sans mon travail sur la sophistique. Pour l'ontologie, il n'y a que le logos, et le logos dit l'être, selon le modèle parménidien. Gorgias, qui vient après Parménide, fait remarquer que l'être, c'est le poème de Parménide qui le fait être. Il n'existe pas sans le logos de Parménide. Donc l'être est un effet de dire. C'est ainsi que je caractérisais la logologie sophistique, qui s'oppose à l'ontologie (le dire de l'être). À partir du moment où l'on prend conscience que l'être est un effet de dire, on comprend que la langue est une performance, que chaque langue est une performance différente. Nous voilà dans Humboldt, dans le romantisme allemand, dans Schleiermacher, et dans la possibilité de la pluralité des langues - même si on en a une plus maternelle que les autres. Même si l'on appartient à une langue et qu'une langue vous appartient, il y a plusieurs langues. Plus d'une langue : c'est une grande, et antique mais non grecque, nouveauté.

F. D.-B. - Je suis tout à fait d'accord avec ce que vous disiez sur l'esprit de Schleiermacher par rapport à l'entreprise $d u$ Vocabulaire et à la traduction. L'expérience de la traduction, ce que l'on découvre en la pratiquant, enseigne d'abord que la traduction n'est pas la saisie de quelque chose qui appartient à l'autre, comme les théories de la communication semblent l'affirmer, mais plutôt de quelque chose qui échappe à soi. Votre Vocabulaire s'assigne, pour reprendre une belle expression d'Antoine Berman, "l'épreuve de l'étranger ": le détour par d'autres langues, d'autres façons de découper le monde. Il en passe donc par la résistance de certains mots à la traduction. Il montre qu' il y a en fait des couches de signification qui se forment, qui ne sont pas directement superposables. Dans la pensée de Berman, cette épreuve de l'étranger a toujours pour visée dernière de ramener au propre, mais dans un détour qui fait que le propre se trouve fécondé, enrichi, revivifié. Si par «propre » nous entendons les philosophies que nous connaissons et pratiquons, qu' effectue à cet égard le geste du détour par l'étranger?

B. C. - D'abord, le détour par l'étranger opéré par le Vocabulaire n'est pas assez étranger. Nous n'avons pas 
considéré les «philosophies » hors Europe : philosophies orientale, chinoise, hindoue. Nous n'avons considéré que les textes qui constituaient déjà notre propre. C'est donc déjà un gros propre, si j'ose dire, et qui ne subit pas en tant que tel l'épreuve de l'étranger ! L'arabe ou l'hébreu n'ont été pris en compte que comme des langues de passage. Nous avons pour l'instant une toute petite épreuve de l'étranger, au sein d'un grand « nous ».

Avec la deuxième partie du travail qui s'opère, il s'agit d'organiser la transposition, la transplantation du dictionnaire dans d'autres langues, donc dans un autre point de vue. C'est encore dans des langues proches, par exemple le roumain, l'espagnol, le portugais, même si j'ai choisi à chaque fois la langue la plus éloignée : par exemple, le portugais du Brésil, langue elle-même malaxée dans son rapport aux langues indigènes, ou encore l'espagnol du Mexique. Je dirais donc que nous sommes en train d'exporter le dictionnaire dans d'autres langues et de voir ce que cela donne quand on le voit d'ailleurs. Même si ce sont des langues proches, il y a déjà un geste à la fois étrange et divers.

Avec la transposition en anglais à Princeton c'est en train de se faire grâce à Emily Apter, Jacques Lezra, Michael Wood -, on est obligé de reformater le dictionnaire : beaucoup de choses restent inchangées, mais l'intention n'est pas tout à fait la même. Il s'agit, comme le dit Jacques Lezra, de se servir de l'English contre le globish, donc aussi de se servir de l'herméneutique contre la philosophie analytique. C'était seulement l'une des idées du dictionnaire, mais pour la transposition en anglais, cela devient l'idée principale, et cela oblige à des reformulations, des simplifications, des complexifications différentes.

En arabe, nous avons choisi de le faire volume par volume. Le premier volume vient de sortir, sur le droit et la loi, dirigé par Ali Benmakhlouf. Dans le cas de l'arabe, le seul fait de la traduction et du choix de tel terme plutôt que tel autre pour traduire droit et loi, relance des questions considérables. Elles ne sont pas explorées comme telles dans le dictionnaire, mais elles le seront à partir de lui.

Enfin, dans un monde encore plus lointain, je commence à m'intéresser aux intraduisibles de la langue chinoise. Je commence à avoir des étudiants chinois qui travaillent sur comment on peut traduire, par exemple, les catégories d'Aristote. D'une certaine manière, on peut dire que c'est la question du Vocabulaire.

F. D.-B. - Si je vous comprends bien, il y a eu une toute petite épreuve de l'étranger, car il est difficile de sortir de nos façons de penser tout en restant à l'intérieur de la philosophie européenne.

B. C. - En effet.

F. D.-B. - François Jullien, par exemple, a des mots assez durs à l'égard de la pensée européenne : il dit qu'elle est incapable de se dessaisir de ses présupposés, de se désarmer. Elle fait même exactement le contraire, puisqu'elle procède par accumulation. Le Vocabulaire aussi procède par accumulation...

B. C. - ... mais par accumulation de problèmes, non pas de certitudes.

F. D.-B. - Donc il ébranle les présupposés, les certitudes, par langues interposées.

B. C. - Je pense que la traduction est vraiment, ô combien, la possibilité d'ébranler les certitudes en philosophie.

F. D.-B. - C'est pour cette raison que vous insistez sur l'emploi du terme de "mots» pour désigner les entrées $d u$ dictionnaire. C'est des mots que l'on part, mais on aboutit aussi à ébranler les concepts.

B. C. - Les mots ébranlent les concepts. C'est toute l'idée du dictionnaire : comprendre qu'on philosophe en langues ébranle la certitude de l'universalité philosophique.

F. D.-B. - En dehors d' une critique d'un certain nombre de clichés courants dans la manière de percevoir le travail philosophique et les langues, l'entreprise implique aussi une critique des universaux.

$B$. C. - Les « universaux » relèvent d'abord d'une doctrine très précise, très particulière, c'est un moment 
doctrinal de l'histoire de la philosophie. Je dirais plutôt qu'il y a une critique de l'universel, qui apparaît comme notre universel (ce « notre » est déjà problématique).

F. D.-B. - C'est donc une entreprise qui ne peut pas se refermer sur elle-même, ni dans le temps, ni à l'intérieur des langues choisies, ni en se repliant sur une exhaustivité de ses entrées. Je le vois un peu comme un organisme vivant, ce Vocabulaire : une sorte de rhizome qui ne peut que croître et s'étendre. On peut même imaginer un texte interactif avec des liens hypertextes dans lequel chaque mot serait mis en rapport avec ses traductions.

B. C. - Absolument. Mais j'ai deux remarques. Je ne voudrais pas qu'on imagine que le Vocabulaire ou moimême nous arrêtons à l'idée que les intraduisibles sont des sémantèmes. Nous savons que la syntaxe est l'arcade des langues, et qu'il n'y a pas de sémantèmes sans syntaxe. Nous en tenons compte autant que nous pouvons. $\mathrm{Si}$ on prend par exemple l'entrée Esti [troisième personne du singulier du verbe grec einai, être, NB], je n'ai eu de cesse que de montrer comment la sémantique et la syntaxe étaient en coprésence. C'est une très mauvaise querelle que de séparer l'un de l'autre.

Pour revenir à votre idée de rhizome, nous avons commencé, grâce à des fonds européens, à modéliser une entrée comme elle pourrait exister sous forme hypertextuelle, c'est-à-dire à la mettre en cartographie avec les autres entrées. Il s'agit de l'entrée Bild. Dans Bild, nous avons montré la profondeur du rapport entre les langues à partir de chaque phrase citée : par exemple une citation de Luther (« Dieu créa l'homme à son image »), que l'on retrouve dans la langue originale, dans les autres langues, dans les différentes traductions. C'est une manière de fabriquer l'héritage culturel européen, de le mettre en cartographie à partir des très grands textes que l'on récupère dans leur problématicité traductive. La deuxième étape de cette plateforme serait de mettre sur le Net l'ensemble des traductions du Dictionnaire, et de comprendre comment on passe d'une langue à l'autre à partir des transformations, omissions, ajouts, y compris des ajouts d'entrées, pour produire le géométral des différences. D'une langue à l'autre, il a fallu changer beaucoup de choses, et pas les mêmes. Par exemple, quand en français il m'arrive de discuter les traductions d'Aristote, ce sont les traductions connues et en usage parmi les étudiants, comme celle de Tricot. Ce ne sont évidemment pas celles qui sont en usage au Brésil, et ce sont d'autres contresens qu'il s'est agi de répertorier, de rendre manifestes. Même pour ces petites choses, qui donnent des aperçus foudroyants sur la manière dont la philosophie est transmise, il nous faut mettre les différences en rapport les unes avec les autres. Le Net permet en quelque sorte d'avoir le point de vue de tous les points de vue, donc le point de vue de Dieu. C'est ce qu'il s'agirait de travailler maintenant.

F. D.-B. - C'est un peu comme si vous faisiez une histoire conjointe des traductions et de la philosophie.

$B$. C. - Oui, et en partant des problèmes contemporains. J'ai omis de préciser, quand vous m'avez demandé comment nous avions choisi les entrées, qu'il fallait que cela nous intéresse aujourd'hui. Cela pose des problèmes aujourd'hui d'intercompréhension entre philosophes. Ce sont des symptômes d'ouverture différente des mondes.

F. D.-B. - On s'en aperçoit par exemple quand on traduit un texte d'un historien d'art, comme ça m'est arrivé, qui fait appel à des passages de Heidegger ou de Hegel qu'il a lus en anglais et dont on doit rechercher la traduction française. Cela devient un problème terrible : on comprend pourquoi il comprend Hegel de cette manière et avec les conclusions qu'il en tire en anglais, mais ça n'a rien à voir avec le français ni avec l'allemand. Or règne toujours, dans l'édition française, cette loi qui veut que si une traduction existe, c'est celle-là qu' on cite.

B. C. - C'est un scandale intellectuel. C'est une histoire de droits, qui relève du juridique et du financier. Il faudrait mettre en branle le droit de la traduction.

F. D.-B. - Dans son livre sur John Donne, Pour une critique des traductions, Antoine Berman insiste sur l'importance des commencements dans une traduction. Il dit à ce propos qu' une première phrase traduite engage une manière spécifique de traduire, que la suite ne fera que dérouler inéluctablement. François Jullien dit à peu 
près la même chose de la genèse de la pensée, dans Entrer dans une pensée : une première phrase engage la suite de la pensée, d'une façon dont on ne pourra plus se déprendre ensuite. Que pensez-vous de ce rapprochement? Correspond-il à votre expérience de traductrice, de lectrice de traductions?

B. C. - Je pense que c'est vrai. La première phrase que l'on écrit d'un texte, que ce soit en traduction ou en pensée, engage toute la suite... sauf quand on la corrige. Nous sommes tous mono-idéiques, nous avons tous eu à un moment donné l'intuition de voir quelque chose d'important, et nous suivons cette intuition ; cela vaut absolument pour François Jullien et Antoine Berman, qui sont très mono-idéiques. J'ai moi aussi cette expérience-là (Dieu sait que je suis mono-idéique, que la sophistique m'a fourni un levier, jusque dans ma manière d'appréhender la traduction - je parlais tout à l'heure de logologie). Mais je pense aussi que les premières phrases se corrigent, se transforment après coup, et qu'on se rend compte que cette première phrase écrite ne voulait pas du tout dire ce qu'on croyait, donc que c'en est une autre... J'ai aussi cette expérience de la métamorphose constante, et de l'incertitude quant à la première phrase. Même si elle est écrite, j'en suis très incertaine. Bref, j'aurais pu rester heideggérienne! Ou devenir bollackienne [rires]. Je crois que le mélange, dans une première phrase, des premières phrases possibles, est quelque chose qu'il faut prendre en considération. Au fond, peut-être que je ne sais pas quelle est vraiment ma première phrase, parce que je ne sais pas si c'est de la philosophie, de la poésie, de la peinture. Et j'en suis très heureuse.

F. D.-B. - C'est vrai quand vous écrivez. Mais quand vous traduisez, la première phrase vous est donnée dans une langue.

B. C. - Pas du tout. Pour Parménide, par exemple, il n'y a pas de première phrase, et il n'y a pas de première phrase sans deuxième phrase qui nous permette de refaire la première phrase. Et ainsi jusqu'à la dernière, avec, simplement, le fait qu'on finit par être fatigué et lassé de revenir sur la première phrase. Mais pour moi, il n'y a pas de première phrase en traduction, ni en pensée, même si le contraire est vrai aussi. J'essaierais donc toujours d'être le moins mono-idéique possible, y compris en traduction : sous chaque première phrase, entendre tous les possibles. En revanche, peut-être pourrais-je substituer à cette expérience de la première phrase une autre expérience, celle de ce qui apparaît quand on travaille. C'est particulièrement clair en peinture : évidemment, tout dépend peut-être des premiers gestes, des premières couleurs que l'on place sur la toile, mais il y a un moment où quelque chose qu'on n'attendait pas du tout apparaît et se voit. Ce moment-là, on peut le suivre. C'est vrai pour moi dans tout : dans la vie, dans la manière d'écrire, de penser, de parler, de peindre. Quelque chose apparaît qu'on n'attendait pas, et à partir de là, on suit. Mais il y a un moment où, quand on suit trop, quand on croit qu'on suit vraiment, on esquinte tout. Il y a donc un suivi très ténu à opérer de ce qu'on n'attendait pas.

\section{F. D.-B. - Et qu' il faut arrêter avant la catastrophe?}

B. C. - C'est peut-être simplement parce que je manque de génie. Si j'étais vraiment peintre, comme l'étaient ma mère et mon père, j'accepterais que ce qui apparaît là se catastrophe, s'abîme, et j'en referais, et je travaillerais cela précisément. Je ne suis pas peintre, donc ce n'est pas comme ça que je procède : quand quelque chose apparaît, je le chouchoute, et j'arrête, avant que ça ne s'abîme. Je suis peut-être philosophe car ce qui apparaît, en philosophie, je veux bien l'abîmer.

F. D.-B. - Quels prolongements verriez-vous au Dictionnaire?

B. C. - L'une des suites possibles du geste du Dictionnaire, ce serait les intraduisibles des trois monothéismes. Réfléchir sur la traduction, ou la non-traduction, des livres sacrés (puisqu'on ne traduit pas le Coran) : comprendre à chaque fois quel est le statut de la traduction, et autour de quels mots chaque livre s'enroule. J'ai commencé à mettre cela sur pied, à la demande du prince Hassan de Jordanie, qui m'avait demandé de réfléchir à une concordance des valeurs. Je lui ai expliqué qu'une concordance des valeurs ne présentait pour moi aucun intérêt : c'est la discordance des mots qui est intéressante. À partir de là, on pourra peut- 
être réfléchir à une éventuelle concordance des valeurs, mais il faut partir de la discordance des langues et des mots. J'ai proposé de travailler sur une vingtaine de mots par texte en langue, autour desquels chaque praticieninterprète pense que le texte sacré s'enroule. C'est un point de départ comparatiste : on peut se demander comment on dit «Dieu », « femme », « croire » et quelles sont toutes les acceptions homonymiques de chacun de ces termes dans une langue donnée, quelles sont les acceptions contextuelles, comment tout cela fonctionne et se discorde.

F. D.-B. - Il s'agit de partir d'une discordance pour arriver à une concordance.

B. C. - Partir d'une discordance des mots pour arriver à la possibilité d'une concordance des valeurs. Comme je ne sais pas ce que veut dire « concordance » ni « valeurs », ce n'est pas trop difficile d'y arriver !

F. D.-B. - Vous voilà investie d'une mission très importante politiquement!

B. C. - Je faisais remarquer qu'il n'était pas indifférent que ce soit une femme, d'origine juive et complètement païenne, baptisée catholique, qui travaille là-dessus ! On a eu quelques réunions avec des amis qui pourraient y participer, cette démarche pourrait faire partie du projet européen que nous voudrions mettre en place.

Entretien transcrit avec la collaboration d'Émeline Durand 